\title{
Téoros
}

Revue de recherche en tourisme

\section{Les réseaux de distribution des voyages aux États-Unis}

\section{Pauline J. Sheldon}

Volume 9, numéro 2, juillet 1990

Le marché américain

URI : https://id.erudit.org/iderudit/1080031ar

DOI : https://doi.org/10.7202/1080031ar

Aller au sommaire du numéro

Éditeur(s)

Université du Québec à Montréal

ISSN

0712-8657 (imprimé)

1923-2705 (numérique)

Découvrir la revue

Citer cet article

Sheldon, P. J. (1990). Les réseaux de distribution des voyages aux États-Unis. Téoros, 9(2), 36-42. https://doi.org/10.7202/1080031ar

\section{Résumé de l'article}

The U.S. Travel distribution network is changing as less traditional intermediaries and more advanced technologies are developed to match suppliers with consumers. Computer networks are likely to become more global, more comprehensive and more accessible to all travel planners making their jobs easier. All travel planners will be presented with new challenges and demands as the travel market diversifies. The nature of the travel product will ensure the existence of travel intermediaries, however, their future success will be dependent on their ability to adapt to change.
Ce document est protégé par la loi sur le droit d'auteur. L'utilisation des services d'Érudit (y compris la reproduction) est assujettie à sa politique d'utilisation que vous pouvez consulter en ligne.

https://apropos.erudit.org/fr/usagers/politique-dutilisation/ 


\section{Les réseaux de diștribution des voyages aux Etats-Unis}

Les canaux de distribution des voyages aux États-Unis deviennent de plus en plus complexes à mesure que les foumisseurs cherchent de nouvelles façons de rejoindre les voyageurs. Ils prennent des formes très diverses, allantd'une opération en uneétape où le consommateur traite directement avec le foumisseur à une opération en deux, trois ou même quatre étapes où il y a plusieurs intermédiaires. Il y a plus de sortes d'intermédiaires dans l'industrie des voyages aux États-Unis qu'auparavant. En plus des agents de voyages et des grossistes en voyages, les intermédiaires incluent les groupeurs, les maisons spécialisées dans les voyages-motivation, les organisateurs de réunions et de congrès, les "courtiers en certificats" (coupon brokers) et les grands magasins. Les réseaux d'ordinateurs jouent aussi un rôle beaucoup plus grand qu'avant. Dans le présent article, nous allons décrire la situation de ces intermédiaireset analyser 1 'orientation que vont prendre les réseaux de distribution des voyages dans les années à venir.

\section{Intermédiaires}

Même si certains fournisseurs ou prestataires vendent directement aux consommateurs sans intermédiaire, la majorité des produits touristiques vendus aux États-Unis sont distribués par l'entremise d'un ou de plus d'un des intermédiaires suivants.

\section{Agents de voyages}

Les agences de voyages représentent le groupe le plus nombreux du secteur de la distribution des voyages. Au début de 1989, on comptait 32497 agents de voyages aux États-Unis, ce qui représente une augmentationde $7,7 \%$ par rapport àl'année précédente ${ }^{(1)} . \mathrm{Si}$ on regarde comment les

Pauline $J$ Sheldon est profegseure en touriame a l'Université d'Hawaï. Ce texte, écrit pour Téoros, a été traduit de l'englais avec l'acoord de l'auteure. agences sont réparties entre les États, on constate que $51 \%$ des agences sont concentrées dans six États, la Californie venant au premier rang de tous les États avec 5093 agences. La majorité des agences de voyages aux États-Unis (près de 92\%) sont de petites agences ayant un chiffre d'affaires annuel au titre des voyages par avion de moins de 3 millions de dol$\operatorname{lars}^{(2)}$ (voir tableau 1). Les agences ayant un seul bureau constituent aussi la majorité $(61,2 \%)$, mais le nombre de grandes agences à plusieurs succursales a augmenté ces dix dernières années en raison d'acquisitions. De 1984 à 1986,3600 agences de voyages ont changé de mains aux États-Unis ${ }^{(3)}$. La plus grosse fusion est peut-être celle qui a eu lieu à la fin de 1989 quand Thomas Cook Travel Inc. a fusionne avec Crimson Travel et Heritage Travel, créant une nouvelle méga-agence qui, avec 327 emplacements, représente un chiffre $d^{\prime}$ affaires annuel au titre des voyages par avion de 1,3 milliard de dollars U.S.. Les principaux buts de ces fusions et de ces acquisitions sont de constituer du volume pour que les agences puissent obtenir de meilleurs taux des foumisseurs, de consolider des comptes de société, ou de profiter au maximum des systèmes de réservations informatisés.

Les ventes totales des agences de voyages par produit sont présentées au tableau 2. La plus grosse partie du volume d'affaires d'une agence de voyages typique (environ $60 \%$ ) provient des voyages par avion, qui représentaient en moyenne par semaine $26424 \$$ (U.S.) par agence en 1984. Les croisières viennent au deuxième rang ${ }^{(5)}$, et les voyages a forfait (qui ne figurent pas dansletableau)représentent $24 \%$ duvolume des agences. Toutefois, beaucoupd'agences commencent maintenant à se spécialiser pour être plus compétitives, de sorte que la répartition du chiffre d'affaires des agences selon les produits vendus varie d'une agence à l'autre. Certaines agences se spécialisent dans les voyages commerciaux, d'autres se spécialisent dans les croisières uniquement, d'autres encore se spécialisent dans les voyages vers certaines destinations. Le tableau 3 montre dans quelle mesure les foumisseurs comptent sur les agences de voyages pour vendre leurs produits. Les organisateurs de croisières et les forfaitistes sont les voyagistes qui dépendent le plus des agents de voyages, tandis que les sociétés ferroviaires et les compagnies d'autocars sont ceux qui dépendent le moins d'eux.

Une enquête menée récemment auprès de 501 propriétaires d'agence de voyages par le groupe Daniel Yankelovich Group (1989) montre que $68 \%$ des agents de voyages prévoient sespécialiser davantage à l'avenir, la moitié d'entre eux environ prévoyant organiser plus de voyages à thème. L'enquête montre aussi que la plupart des agents de voyagessont maintenant abonnés au service 800 pour mieux servir leur clientèle et qu'ils prévoient aussi recourir davantage à la stratégie du rábais à l'avenir.

Les agents de voyages doivent déjà rivaliser avec d'autres concurrents et cette concurrence devraits 'intensifierencore plus dans les annés à venir. Ces pressions donnent à penser que les agents de voyages doivent accorder une attention toute particulière à leur rôle de conseiller s'ils veulent continuer d'avoir du succès au milieu des nouvelles techniques de vente et des progrès technologiques. Des points de vente au détail traditionnels comme les magasins d'alimentation et les grands magasins ont commencé à vendre des voyages. Par exemple, Sears Travel Network se prépare à travailler avec National Leisure Group à vendre des voyages d'agrément par l'entremise de ses magasins, de numéros de téléphone sans frais et de catalogues.

L'utilisation accrue d'ordinateurs personnels à la maison et au bureau représente aussi une source de concurrence potentielle pour les agents de voyages. Les voyageurs peuvent vérifier la possibilité d'obtenir des produits touristiques et faire des réservations en s'abonnant à un réseau comme Compuserve, Genie ou The Source. Ces réseaux donnent accès à des bases de données touristiques comme la version Electronic Edition du OfficialAirline Guide, Easy Sabre (une version conviviale et pilotée par menus du système de réservations Sabre), Travel Shopper de la compagnie 
TWA et le système Advance d'American Express. Jusqu'ici, l'accès à ces réseaux n'a pas beaucoup empiété sur le rôle des agents de voyages. Les principaux. utilisateurs de ces réseaux sont les voyageurs d'affaires, les grosutilisateursd'ordinateurs (qui les utilisent souvent comme des babillards pour faire part de leurs commentaires sur les produits touristiques) et les consommateurs qui préferent tout simplement faire les choses eux-mêmes. Comme leur utilisation nécessite une dépense de temps et d'argent par le consommateur pour accomplir ce qu'un agent de voyages foumit gratuitement, cela n'est pas surprenant.

Les distributeurs automatiques de billets sont un autre usurpateur potentiel des fonctions de l'agent de voyages; on les trouve maintenant dans la plupart des aéroports de centres urbains aux États-Unis. Ces machines permettent aux passagers de faireleurs réservations eux-mêmes de façon indépendante, d'obtenir leurs billets, de recevoir la confirmation de leurs places et d'obtenir leurs cartes d'embarquement, de mettre à jour le nombre de milles qu'ils ont accumulés comme grands voyageurs, et de tout facturer sur leurcarte de crédit. Encore ici, les voyageurs d'affaires sont les principaux utilisateurs. Certains transporteurs ont abandonne ces machines parce qu'elles n'étaient pas assez utilisées.

Les agences qui se spécialisent dans les entreprises utilisent aussi des imprimantes de billets satellites (IBS) pour ameliorer leur compétitivité. Les IBS permettent d'imprimer les billets, les itinéraires et les cartes d'embarquement au bureau même du client (ou à un autre endroit), tout en permettant à l'agent de voyages d'exercer un contrôle central à l'intérieur de son propre système informatique. À l'heure actuelle, 6,6\% des agences aux Êtats-Unis utilisent des IBS et plus de 2000IBS ontété installées jusqu'ici dans des entreprises. Des sociétés indépendantes autres que des agences de voyages commencent maintenant à installer des IBS dans des hôtelset àd'autresendroits. Par exemple, la société Hoteleticket met à la disposition des voyageurs un système permettant de faire des changements de dernière minute àl'aide d'une IBS installée dans un hôtel. Le San Francisco Parc 55 Hotel a une IBS qui est relíé à tous les systèmes de resservation des compagnies aériennes. Entre autres sociétés prévoyant installer des réseaux indépendants d'IBS, mentionnons la sociéte Airline Computerized Ticketing, Q-DAT et Western Union. Elles font payer des frais de service aux agences de voyages qui utilisent les
TABLEAU 1

Volume des ventes des agences de voyages

Moins de $300000 \$$
$300000 \$-500000 \$$
$500000 \$-1000000 \$$
$1000000 \$=3000000 \$$
$3000000 \$-5000000 \$$
$5000000 \$-10000000 \$$
$10000000 \$$ et plus

Ventes annuelles selon la ARC
Pourcentage des agences
16,3
13,5
29,1
32,6
4,6
2,6
1,3

Source: Travel Weekly, 11 septembre 1989 , page 8.

Le volume des ventes est exprimé en dollars amerricains (U.S.).

\begin{tabular}{lccc} 
& \multicolumn{4}{c}{ TABLEAU 2 } \\
Ventes des agences de voyages aux États-Unis \\
par produit, 1983-1987
\end{tabular}

\begin{tabular}{|ll}
\hline & \multicolumn{1}{c}{$\begin{array}{c}\text { TABLEAU 3 } \\
\text { Dépendance des fournisseurs à l'endroit } \\
\text { des agents de voyages aux Êtats-Unis }\end{array}$} \\
& $\begin{array}{ll}\text { Pourcentage estimatif du volume } \\
\text { réservé par les agents }\end{array}$ \\
\hline Compagnies d'aviation & $70 \%$ (vols intérieurs) \\
& $80 \%$ (vols internationaux) \\
Hờtels & $25 \%$ (aux États-Unis) \\
& $85 \%$ (a l'étranger) \\
Compagnies de croisière & $95 \%$ \\
Sociétés ferroviaires & $37 \%$ \\
Compagnies d'autocars & Moins de $10 \%$ \\
Location d'automobiles & $50 \%$ \\
Voyages à forfait & $90 \%$ \\
\hline
\end{tabular}

Source: Waters, Somerset, Travel Industry World Yearbook, 1989. 
machines, mais elles prévoient aussi leur donner une commission sur les ventes. Certains agents de voyages $s^{y}$ opposent a l'installation d'IBS par des sociétés indépendantes parce qu'ils craignent d'être contournes. La Airline Reporting Corporation (ARC), toutefois, réglemente l'utilisation des IBS et approuve tous les emplacements.

\section{Les groupeurs}

Les groupeurs sont des agents pour les sociétés aériennes qui vendent un surplus de billets sur des vols intemationaux vers

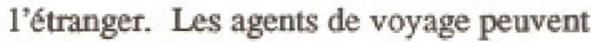
offrir des tarifs plus avantageux a leurs clients en ayant recours à des groupeurs qu'en réservant directement auprès de la société aérienne. Le groupeur garantit à la société aérienne un volume annuel vers une destination donnée et vend les sièges par l'entremise d'agents de voyage en leur donnant des commissions de $15 \%$ a $50 \%{ }^{(6)}$. Onestimeque les groupeurs peuvent vendre jusqu'a $80 \%$ des sièges d'une société aérienne, surtout sur les routes à rabais très élevé des États-Unis vers 1'Orient. Ilexiste au moins 25 groupeurs aux États-Unis, mais aucune association nationale ne met de l'avant leur professionnalisme ou $\mathbf{n}$ 'applique un code de déontologie. Bien que les groupeurs jouent un rôle important dans le réseau de distribution des voyages, certains organisateurs de voyages craignent qu'ils ne grignotent leur secteur d'activité. Les consommateurs qui avaient 1 'habitude d'acheter un forfait peu coûteux peuvent maintenant béneficier de tarifs similaires auprìs d'un agent de voyages en faisant appel à un groupeur.

\section{Les "courtiers en certificats" et les programmes "grands voyageurs"}

Inaugurés par American Airlines en 1981, les programmes "grands voyageurs" sont maintenant courants dans 1 'industrie des voyages aux États-Unis. Par ces programmes, les sociétés aériennes voulaient que les gens se limitent à une marque, mais aujourd'hui, de nombreux voyageurs sont membres de plus d'un programme. En 1988, la plupart des sociétés aériennes accordaient, en prime, le triple des milles parcourus; cela a entraîné des pertes énormes pour les sociétes aériennes évaluées à 1,24 milliard de dollars. Depuis, la plupart des transporteurs estampillent une date limite pour restreindre 1'utilisation des primes. Malgré leur succès, seulement $30 \%$ des primes-voyages sont utilisées $(?$, peut-être parce que certains voyageurs accumulent trop peu de milles pour pouvoir les échanger pour des primes ou que ceux qui en accumulent assez n'ont pas le temps ou ledésird'utiliserleurs primes ou désirent voyager en périodes d'interdiction.

Durant la demière décennie, un nouvel intermediaire est apparu dans 1'industrie des voyages: ce sont les "courtiers en certificats". Ces courtiers vendent les primes-voyages des voyageurs qui ne désirent pas s'en servir eux-mëmes. Ils ont fait leur apparition en 1979 en regard des certificats d'United Airlines et d'Eastern Airlines. Étant donné qu'il est interdit de vendre les primes-voyages dans la plupart des programmes, les "courtiers en certificats" font actuellement l'objet d'enquêtes. Bon nombre d'entre eux ont fait faillite et les sociétés aériennes ont intenté des poursuites contre une douzaine d'entre eux.

\section{Les organisateurs de réunions, de congrès et de voyages-motivation}

Le nombre de réunions et de congrès ne cesse d'augmenter aux États-Unis et les organismes spécialisés dans l'organisation de voyages pour les groupes qui y assistent sont aujourd'hui une composante significative du réseau de distribution des voyages. Depuis les années '60, les voyages-motivation représentent un pourcentage élevé de ces voyages. Il s'agit de voyages qu'une société paie pour récompenser ses représentants, ses distributeurs, ses vendeurs, ses clients ou d'autres employés. On trouve une centaine d'agences de voyages-motivation aux ÉtatsUnis; certaines se spécialisent dans les voyages de luxe de petits groupes de voyageursetd'autres, dans desdestinations enparticulier. Carlson Marketing Group et Maritz Inc. sont deux des plus importantes agences de voyages-motivation aux ÉtatsUnis, mais d'autres agences de voyages importantes, comme Woodside Travel et Ask Mr. Foster Travel Service ont aussi leurs propres services de voyagesmotivation. La Society of Incentive Travel Executives (SITE), crée en 1973, exige que ses 1200 membres répartis dans 42 pays respectent un code de déontologie ${ }^{(\mathrm{s})}$.

Le montant total consacré aux voyagesmotivation aux États-Unis a augmenté de 50 millions de dollars en 1960 à près de 2 milliards de dollars en 1986. D'après une enquéte de la STTE, environ $20 \%$ des voyages-motivation sont réservés auprès d'agences de voyages-motivation, $59 \%$ sont réservés directement par le service des voyages de 1'entreprise, $48 \%$ font appel à un agent de voyages local et $13 \%$ sont réservés directement auprès de voyagistes. (Lasomme des pourcentages dépasse $100 \%$ parce que certaines sociétés peuvent utiliser plus d'une façon de faire des réservations) $)^{(9)}$.

\section{Les organisateurs de voyages}

En 1986, seulement $4 \%$ des voyages faits aux États-Unis étaient des forfaits etenviron $27 \%$ des Américains qui se sont rendus a l'étranger ont acheté des forfaits ${ }^{(10)}$. Malgré le passage des voyages en groupe aux forfaits individuels, les organisateurs de voyages (aussi dénommés voyagistes ou tours-operateurs) et les grossistes en voyages sont restés des intervenants importants dans le reseau de distribution des voyages aux Etats-Unis. En effet, ils répondent aux désirs des voyageurs en regard des forfaits individuels en offrant des produits adaptables qui permettent aux consommateurs de concevoir eux-mêmes leurs voyages à partir de diverses composantes. Lacompétitionqui confronte les organisateurs de voyages vient de plusieurs côtés. Actuellement, environ $20 \%$ des agents de voyages des Etats-Unis organisent eux-mêmes leurs tours ${ }^{(11)}$. Les sociétés aériennes et les autres foumisseurs organisent et vendent eux aussileurs propres tours.

Les organisateurs de voyages se divisent en deux groupes. Il y a d'abord les grandes sociétésqui vendent une quantité importante de tours avant tout internationaux. Environ 45 d'entre elles sont membres de l'United States Tour Operator Association (USTOA), une association d'organisateurs de voyages dont l'objectif principal consiste à établir des nomes professionnelles au sein de 1 'industrie. Les agents de voyages preferent faire des reservations auprès de ces sociétés parce qu'elles sont cautionnées et stables. Mais, depuis 1987, à cause de la forte concurrence sur le marché, quelques sociétes membres de 1'USTOA ont fait faillite. Il y aensuite les petits organisateurs de voyages qui offrent des tours à intérêt spécial ou par autocar. On en compte environ 1000 regroupés dans la National Tour Association (NTA).

Les brochures sur les tours constituent le principal instrument de vente des organisateurs de voyages et ils recourent surtout aux agents de voyages. La totalité des tours organisés par les sociétés membres de l'USTOA et $75 \%$ des tours organisés par les membres de la $N T A$ sont vendus par des 
agents de voyages. Les organisateurs de voyages qui ne sont pas membres des deux associations précitées font beaucoup plus affaires directement avec le consommateur parce que les agents de voyages sont moins portés à vendre leurs tours. $D^{\prime}$ après une enquête sur les organisateurs de voyages dont les résultats ont été publiés dans Tour and Travel News, 57,4\% d'entre eux font affaires directement avec les consommateurs. Désirant protéger les agents de voyages contre la vente de produits peu recommandables, $1^{\prime}$ American Society of Travel Agents (ASTA) a mis au point un plan de protection qui exige des organisateurs de tours qui vendent par l'entremise des membres de l'ASTA de remettre un cautionnement de $100000 \$$ comme assurance. Un nombre de plus en plus grand de clients désirent maintenant faire des réservations à la demière minute; les organisateurs de voyages ont donc recours à des entreprises de messageries pour satisfaire cette demande.

\section{Les systèmes de réservation informatisés (SRI)}

De tous les développements à survenir dans les reseaux de distribution des voyages, les systèmes de réservation informatisés (SRI) ont eu le plus de répercussions. Les sociétés áriennes ontété les premières à informatiser les réseaux de distribution de voyages et les autres organismes (les hôtels, lesentreprises de location d'automobiles et les organisateurs de tours et de croisières) leur ont emboitté le pas. Les premiers systèmes de réservations informatisés ont été mis sur pied vers la fin des années '60 par des sociétés aériennes qui s'en servaient pour leurs besoins intemes. Leur valeur pour les agents de voyages est vite devenue evidente, et, en 1976, ils ont été installés dans les bureaux des agents de voyages. Depuis, des systèmes de réservation de voyages par avion se trouvent dans les bureaux de tous les organisateurs de voyages et ils se sont développés de façon à renfermer des renseignements sur toutes les facettes d'un voyage. En fait, il serait plus approprié de les appeler des systemes d'information sur l'industrie des voyages.

Les cinq systèmes de réservations aêriennes dont disposent les agences de voyages des Etats-Unis depuis un certain nombre d'années sont Sabre (American), Apollo (Covia/United), SystemOne (Texas Air/ Eastem), PARS (Northwest/TWA)et Datas II (Delta). La figure 1 montre le nombre de terminaux pour chaque système en 1988. Au moment où nous écrivons ces lignes,
Datas II et PARS ont entrepris des demarches de fusion pour former un seul système de réservation. La fusion a êté approuvée par le département de la Justice, peut-être parce qu'ensemble, ces deux systèmes ne représentent qu'un faible pourcentage du marche des agents de voyages (ils détiennent $23 \%$ des terminaux. des agences de voyages des Êtats-Unis). En 1989, Datas II et Sabre ont voulu fusionner, mais le département des Transports s'y est opposé parce que leur part du marché des agences de voyages aurait atteint $43 \%$. D'autres vendeurs de SRI, surtout SystemOne et PARS, y étaient fortement opposés et les procureurs du département de la Justice craignaient que la fusion nuise a la concurrence et fasse augmenter les tarifs aériens sur certaines routes. Les SRI ont fait faire des profits enormes à leurs sociétés mères et, depuis la déréglementation, les sociétés aériennes dotées de SRI sont celles qui semblent avoir connu le plus de succès. Le tableau 4 montre les recettes moyennes générées par chaque système, par terminal et par bureau informatise. Dans certains cas, les activités relatives aux SRI sont plus rentables que les activités de transport aérien, et des sociétés distinctes ont été mises sur pied pour les administrer. Le système Sabre est utilisé depuis 1986 par la société AMR, une filiale d'American Airlines, et le systeme Apollo est exploité par Covia, une société a portefeuille pour UnitedAirlinesetd'autres societes qui organisent des voyages. Les SRI sont de plus indirectement responsables de certaines des fusions de sociétés aériennes qui sont survenues ces dernières années. Lorsque la société EasternAirlines a acheté Texas Air en 1987, le système de réservation SystemOne était un élement déterminant de la transaction, tout commel'aété le système PARS lorsque la société Northwest Airlines a achete $T W A$.

En 1984, le département des Transports a mis de l'avant des règles pour régir les rapports entre les agents de voyages et les sociétés aériennes qui vendent des SRI. Une de ces règles visait à éliminer tout artifice des renseignements affiches sur les ecrans SRI. La même année, American Airlines et United Airlines ont été poursuivies par 11 autres sociétés aériennes qui prétendaient qu'elles utilisaient des moyens frauduleux dans leurs systemes. D'autres règles du département des Transports stipulaient la durée maximale (5 ans) d'un contrat entre le vendeur du SRI et

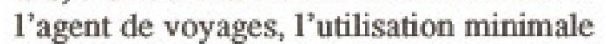
du système requise des agents, et les changements à apporter à la clause des dommages-intérêts liquidés applicable lorsqu'un agent de voyages ne respecte pas un contrat. De nombreux cas de dommagesintérêts liquidés sont maintenant devant les tribunaux parce que des agents de voyages qui ont adopté un autre système refusent de payer ces dommages-intérêts. Dans la plupart des cas, les tribunaux semblent favoriser les vendeurs de SRI.

Toutes ces règles, qui seront en vigueur jusqu" à la fin de 1990 , font actuellement l'objet d'une étude par le département des Transports. Les sociétés qui exploitent les systèmes Sabre et Apollo (qui sont les deux plus importants) espèrent que les règles vont rester inchangées pour pouvoir conserver leurpart du marché, mais les plus petits vendeurs espèrent des changements. qui leur permettront d'accrôtre leur part de marché des agences de voyages. L'ASTA $^{\prime}$ est en faveur d'une durée de trois ans pour les contrats au lieu de la durce actuelle de cinq ans. Le Congrèssepenche actuellement sur les moyens à utiliser pour améliorer la concurrence entre les vendeurs de SRI. Voici certaines des possibilites qui sont a 1'étude: 1) fixer une limite supérieure aux clauses des dommages-intérêts liquides; 2) enlever les SRI aux sociétés aériennes et en faire des sociétés indépendantes sans lien avec les sociétes aériennes; et 3 ) isoler les SRI des sociétés aériennes et, dans l'intérêt du public, en faire un seul système ${ }^{(12)}$. La société System $O$ ne demande également aux vendeurs de SRI des États-Unis d'adopter un code d'éthique semblable à celui qu'on trouve dans les systèmes européens. II faudrait alors que tous les vendeurs affichent d'abord tous les vols sans escale, puis les vols directs, et, ensuite, les correspondances.

Les vendeurs de SRI étendent maintenant leurs réseaux en Asie et en Europe pour mettre sur pied des SRI mondiaux. La figure 3 montre les liens qui existent entre les divers systèmes. (La fusion Datas III PARS n'y apparaît pas, étant donné qu'elle ne s'est pasencore concrétisée). Le logiciel de SystemOne est la base du système européen Amadeus, le système Galileo appartienten partie a Covia, et la technologie du système PARS sert de base au système asiatique Abacts. La technologie du système Sabre a conclu une entente avec les lignes aériennes japonaises dans le but de partager leurs bases de donnes et de travailler de concert à la mise au point de logiciels. A cause de cette mondialisation, les utilisateurs de SRI aux États-Unis vont avoir accès a un nombre beaucoup plus grand de bases de données mondiales sur les voyages.

L'exhaustivite et la pertinence des 
renseignements contenus dans les produits vendus par l'entremise des systèmes de réservations aériennes continuent de $s$ 'améliorer. $\mathrm{La}$ figure 4 montre le pourcentage des réservations de divers produits faites au moyen des systèmes. Bien que les forfaits et les croisières soient ce qui est réservé le moins souvent au moyen d'un SRI, des efforts sont faits actuellement pouren augmenter le nombre.

Des réservations en directet des commandes par brochure sont maintenant offertes dans la plupart des systèmes de réservations aérienne. Un certain nombre de bases de données surles tours peuventêtre consultées par l'entremise des SRI. La société WorldCom Net a mis au point une base de données de ce genre destinée aux organisateurs de voyages et qui s'appelle Tourinc; les abonnés à Apollo y ont accès et, depuis quelques temps, les abonnés à SystemOne aussi. Les abonnés à Apollo ont également accès en direct à un programme appele Automated Tour Operator Package (ATOP) etils peuvent y faire des réservations à même le stock de tours et de croisières; ce programme a été mis au point par une société appelée Autofile. La société World Associates est en train de mettre au point une base de données (appelée World-File) sur divers tours internationaux. Les programmes CruiseMatch et Official Recreation Guide (ORG) sont également disponibles parl'entremise de certains SRI. L'ORG est un système de réservation de voyages d'agrément où figurent les produits des organisateurs de voyages et diverses activités de loisirs et divers événements partout dans le monde.

L'accès, par les SRI, au stock des tours oblige les organisateurs de voyages à tenir un inventaire double, un dans leur propre ordinateur et un autre dans le SRI que la société aérienne met à jour de temps à autre. Les agents de voyages semblent toutefois hésiter à recourir aux SRI pour réserver des tours, mais ils s'en servent d'emblée pour connaître les disponibilités. C'est peut-êttre à cause du fait que les critères de recherche de la plupart des SRI n'aident pas à choisir un tour. C'est loin d'être l'idéal et ne renseigne pas l'agent surles dernières places disponibles. C'est pour cette raison que certains organisateurs de voyages et des societés indépendantes ont mis au point leurs propres systèmes que 1'agent de voyages peut consulter sans passer par un SRI. Par exemple, le système Voyager permet aux organisateurs de voyages d'intégrer leur inventaire de voyages à l'ordinateur central ou de mettre en réseau leur propre ordinateuret l'ordinateur central.

FIGURE 1

Nombre de terminaux de système de réservations aux Etats-Unis, 1988

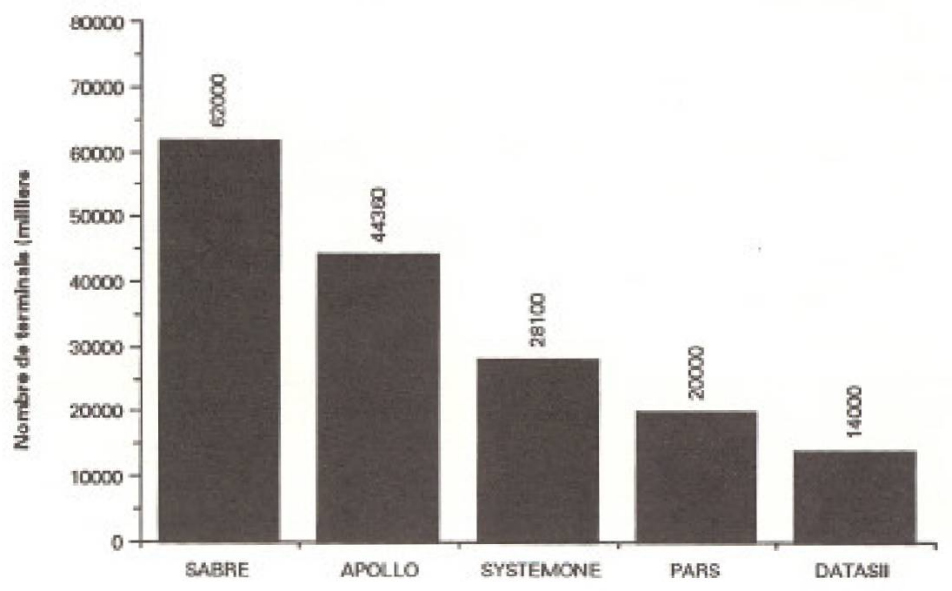

Source: Louis Harris, 1988 Survey.

\section{FIGURE 2}

Pourcentage des réservations faites au moyen des systèmes de réservations informatisés (SRI)

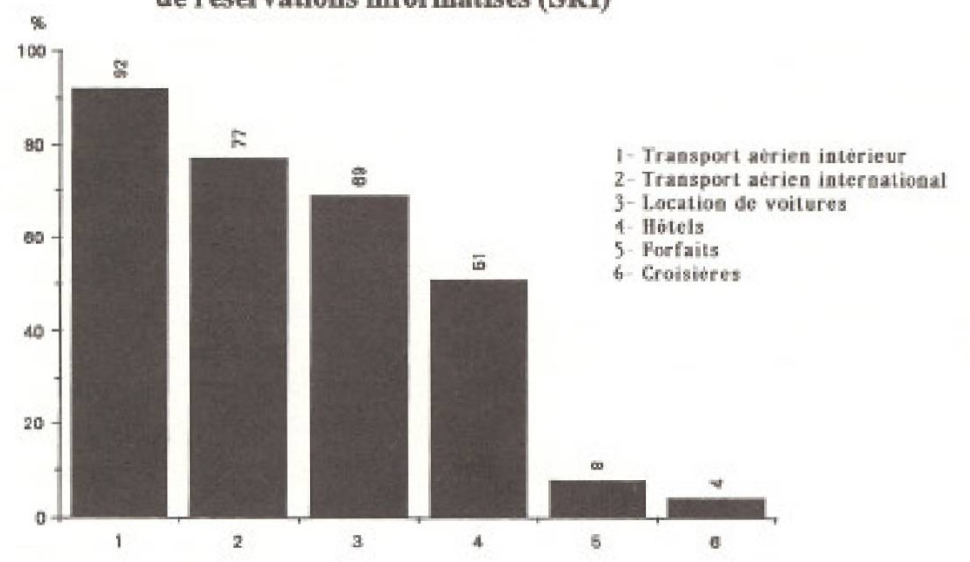

Source: Louis Harris, 1988 Survey.

Ce système comprend 16 critères de recherche différents mieux adaptés au choix d'un tour.

Les liens d'accès direct qui existent entre de nombreux ordinateurs de l'industrie des voyages permettent de renseigner les agents de voyages, par l'entremise de terminaux de SRI, sur les demières places disponibles en regard d'un plus grand nombre de produits. A cause de trouvailles comme Ultraswitch des réservations de chambres d'hôtel peuvent être faites au moyen des SRI et leurs confirmationssont immédiates. Le système Ultraswitch est un réseau informatisé de réservations de chambres d'hôtel qui relie entre eux les ordinateurs de réservations des grandes chaînes hôtelières et le SRI de la société aérienne. Il a êté mis au point par Rupert Murdoch qui l'a vendu dernièrement à Reed International. Le Reed Travel Group est également propriétaire du système de réservation Utell pour les hôtels indépendants. La société Covia est à mettre au point un nouveau système, appelé Covia Reserve, qui permettra aux abonnés d'avoir accès aux réseaux mondiaux de réservation de chambres d'hôtel et de recevoir des confimations en 15 a 20 secondes. Les sociétés de location d'automobiles sont en train de relier, de la même façon que les hôtels, leurs ordinateurs de réservations aux systèmes des sociétés aériennes.

Récemment, certains vendeurs de SRI ont perfectionné la technologie de l'imagerie laser qui permet aux agents d'obtenir des 
Figure 3

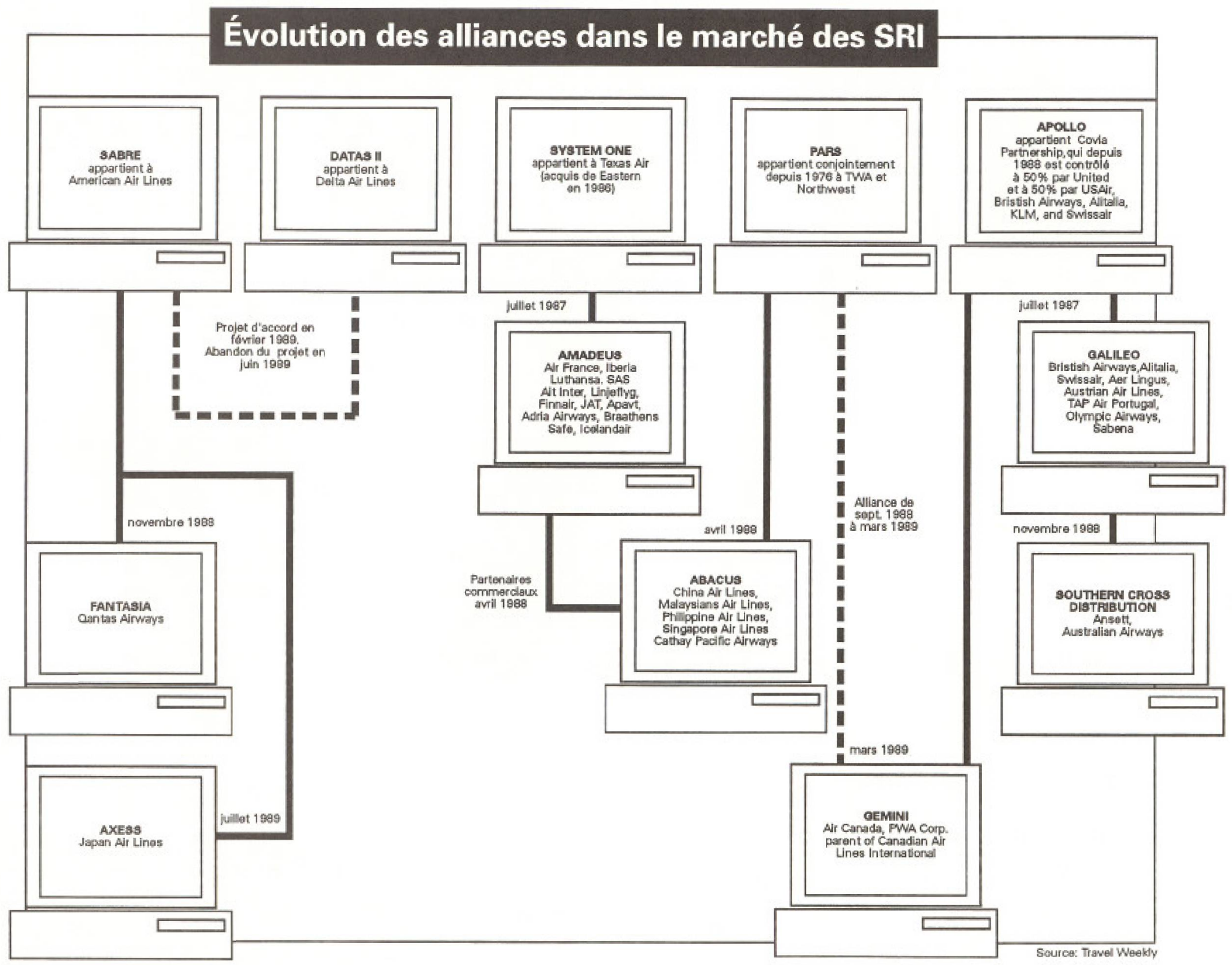


images de leurs produits sur leurs écrans. Le plus perfectionné de ces systèmes serait le système ISIS qui a été mis au point par la sociéte PARS. Des images d'hôtels, d'endroits de villégiature et d'autres produits de voyage sont stockées sur des disques lasers ou sur des CD-ROM et elles peuvent être affichées sur l'écran du SRI. La société Sabre est à mettre au point un produit semblable appelé Sabrevision avec l'aide du Reed Travel Group; ce produit sera surtout axé sur les hôtels et sur les croisières. Il pourra être utilisé sur des ordinateurs personnels et dans le reseau local de l'agence de voyages et un second écran ne sera pas requis pour afficher les images. Delta Airlines a temporairement mis de côté son produit de même nature, appelé DeltaStarView, pour en evaluer les possibilités. Des images mobiles devraient être ajoutées à ces systèmes dans quelques annees.

Des projets de recherche sont en cours pour ameliorer tous les SRI. Un des aspects de base à ameliorer se situerait au niveau des possibilités en regard des tarifs. Les chercheurs sont à mettre au point diverses caractéristiques, comme la notification automatique de nouveaux tarifs moins clevés après qu'un passager a fait une réservation. D'autres aspects à l'étude sont le courrier électronique entre les agents et leurs clients et la possibilité de transférer des données d'unteminal du systeme Sabre à un telecopieur.

\section{Summary}

The U.S. Travel distribution network is changing as less traditional intermediaries and more advanced technologies are developed to match suppliers with consumers. Computer networks are likely to become more global, more comprehensive and more accessible to all travel planners making their jobs easier. All travel planners will be presented with new challenges and demands as the travel market diversifies. The nature of the travel product will ensure the existence of travel intermediaries, however, their future success will be dependent on their ability to adapt to change.

\section{NOTES}

(1) WATERS, Somerset, Travel Industry World Yearbooke: The Big Pleture, 1969, P. 127

(2) Travel Weskly, 11 septembre 1989, p. 8.

(3) FROMMER, Lawrence Jo, How Traval Agencies are Bought and Sold, ASTA Travel News, avril 1986, p. 27.

(4) WATERS, Somerset, Travel Industry World Yoarbook: The Big Picturs, 1989, p. 127.

(5) DAVIDOFF, P, et D., Thavel Febaliting in The U.S.A. Travel and Tourlam.Analyat, dGcembre 1966, pp, 30-38.

(6) ABERNATHY, Melisea, Despite High Riska, Use of Consolidators for International Travel Soars, Corporate Travel, septembre 1988, pp. 16-17.

(7) PETERSON, Randy, Offlelal Frequent Flyer Guldebook, Bonus Books, 1989 .

(8) BREDEMEIEF, Judy, incentive Travelin The U.S.A., dans Travel and Tourism Analyat, septembre 1986 , pp. 25-36.

(9) SOCIETY OF INCENTIVE TRAVEL EXECUTIVES, Incentive Travel: Fact Sheot and Overvlow, juillet 1985 ,

(10) SHELDON, Pauline d, The US. Tour Opevator Industry, dane Travel and Tourlem Analyst, no 3, 1988.

(11) FIELD, Mike, Going Wholesale, Travel Wookly's Loule Harrle Survey, 29 juin 1988.

(12) LEISER, Rioland, Lawmakers Ponder Options to Inonose Competition Among Reservation Syatems, Travel Agent Magazine, 9 octobre 1989, p. 20.

\section{BIBUOGRAPHIE}

BREDEMEIER, Judi, Incentive Travel in The USA dans Travel and Tourkm Analyat, septembre 1986, pp. 25-36.

DANIEL. YANKELONICH GROUP, Looking Toward The Nineties in The Leisure Travel linduatry, Tour and Travel Newse octobre 1989.

DAVIDOFF, Phil et Doris, Travel Retaling in The U.S.A. dans Travel and Tourlom Anshyat, décembre 1986.

SHELDON, Pauline J., The U.S. Tour Operator Industry, dana Travel and Tourhem Analyst, no 3, 1988.

WATERS, Somerset, The Travel Industry World Yoarbook: The Big Pleture, 1989 